\title{
Motor versus Sensory Nerve Conduction Monitoring of Median Nerve during Carpal Tunnel Surgery
}

\author{
Adel R. Al Melisy ${ }^{*}$, Hanan M. El Saadany² \\ ${ }^{1}$ Departments of Neurosurgery, Al-Azhar University, Cairo, Egypt \\ ${ }^{2}$ Department of Physical Medicine and Rheumatology, Tanta University, Tanta, Egypt \\ Email: *adelalmelesy@gmail.com
}

How to cite this paper: Al Melisy, A.R. and El Saadany, H.M. (2021) Motor versus Sensory Nerve Conduction Monitoring of Median Nerve during Carpal Tunnel Surgery. Open Journal of Modern Neurosurgery, 11, 114-121.

https://doi.org/10.4236/ojmn.2021.112014

Received: February 28, 2021

Accepted: April 26, 2021

Published: April 29, 2021

Copyright () 2021 by author(s) and Scientific Research Publishing Inc. This work is licensed under the Creative Commons Attribution International License (CC BY 4.0).

http://creativecommons.org/licenses/by/4.0/

\begin{abstract}
Background/Aim: Carpal tunnel syndrome (CTS) is one of the most common compressive, canalicular neuropathies of the upper extremities, causing hand pain and impaired function. This clinical study was designed to compare the intraoperative median nerve distal motor latency (DML) versus the distal sensory latency (DSL) and sensory nerve conduction velocity (SNCV) during surgical treatment of CTS. Patients and Methods: A total number of 36 wrists in 30 patients with CTS diagnosed by preoperative median MNCS who underwent surgical intervention for median nerve release. Intraoperative measurements of motor distal latency (DML) and proximal latency, amplitude, and conduction velocity and distal sensory latency (DSL), amplitude, and conduction velocity of both the median and ulnar nerves before and after division of the flexor retinaculum (FR). Results: A significant decrease of the intraoperative median nerve distal motor latency (DML) after release $(8.89 \pm$ $0.93)$ in comparison with the preoperative median nerve DML $(6.24 \pm 1.06)$ with $\left(\mathrm{p}<0.001^{*}\right)$. But intraoperative distal sensory latency (DSL) and sensory nerve conduction velocity $(\mathrm{SNCVM})$ show non-significant decrease $(\mathrm{p}=$ 0.161 ). Conclusion: Intraoperative monitoring of median nerve show rapidly improve Intraoperative motor nerve conduction than the sensory nerve conduction after release the FR during carpal tunnel surgery.
\end{abstract}

\section{Keywords}

Flexor Retinaculum, Transverse Carpal Ligament, Carpal Tunnel Syndrome, Median Nerve Conduction Study

\section{Introduction}

Carpal tunnel syndrome (CTS) is one of the most common Entrapment neuro- 
pathy syndromes of the upper limb [1] [2]. Its incidence is 139/100,000 per year for men and 506/100,000 per year for women [3]. Carpal tunnel syndrome (CTS) occurs at a rate of 5.2 per 10,000 full-time workers, and this syndrome requires the longest re-occupational period of all conditions that result in lost work days, with a median of 30 days lost [4]. It arises from entrapment of the median nerve at the wrist; typically the disorder is characterized by numbness or tingling in the sensory distribution of the median nerve in the hand, and may be associated with pain and or weakness of the muscles of the thumb [5]. Women between the ages of 45 - 60 years suffer from CTS more than men with a ratio of 3:1 [6]. CTS often results from repetitive strain in manual jobs but it is also associated with conditions like rheumatoid arthritis, pregnancy due to water retention [7], and diabetes mellitus, which increases the likelihood of a symptomatic response in an already compressed nerve [8]. Conservative treatment with physiotherapy, wrist splints, corticosteroid injections, diuretics, vitamin B6 is proposed initially, whereas surgical treatment is reserved for more severe cases with thenar muscle atrophy or after failure of conservative treatment [9]. Treatments include: splinting, steroids injected locally and surgical release of the transverse carpal ligament. Chronic compression by the TCL leads to nerve swelling proximal and distal to the carpal tunnel. Once that compression is relieved, the nerve should return to baseline size. Whereas EDS are often used to track nerve function, often they do not return to baseline standards after intervention [10].

\section{Aim of the Work}

The objective of this study is to compare the intraoperative median nerve distal motor latency (DML) versus the distal sensory latency (DSL) and sensory nerve conduction velocity (SNCV) during surgical treatment of CTS.

\section{Patients \& Methods}

An analytical prospective study conducted on 36 wrists in 30 patients (6 cases bilateral) with carpal tunnel syndrome (CTS) who had failed conservative treatment and were scheduled for surgical intervention in AL Moasah hospital Tanta from July 2016 to December 2020. Patients diagnosed by history, clinical examination and preoperative nerve conduction study. Follow up of patients done clinically and electro-diagnostically over 6 weeks. Ethical approval and informed consent were obtained from all patients. Twenty three patients were women and 7 patients were men, their age ranged from 25 - 54 years (mean age $39.5 \pm 2.3$ year). All the patients underwent intraoperative electro-diagnostic study (EDS) performed on all participants using the Neuropack, (NIHON KOHDEN System, Tokyo, Japan). All tests were done under similar temperature conditions in the same room. The routine NCS of the upper extremities was performed on each participant. Motor distal and proximal latency, amplitude, and conduction velocity of the median and ulnar nerves, and distal sensory latency (DSL), amplitude, and conduction velocity of both the median and ulnar nerves were meas- 
ured. The motor median NCS was performed with bipolar percutaneous electrodes using standard techniques of supramaximal stimulation. The DML was measured with an active recording electrode placed close to the motor point of the abductor policies brevis and reference electrode $3 \mathrm{~cm}$ distal at first metacarpophalangeal joint. The nerve was stimulated using bipolar stimulation electrodes, with the cathode positioned $3 \mathrm{~cm}$ proximal to the distal wrist crease and at elbow near the volar crease of brachial pulse. The cathode was placed closest to the recording electrode. DSL measure by orthodromic stimulation. For stimulation ring electrodes were placed at $2^{\text {nd }}$ digit. Electrophysiological test results considered diagnostic of severe CTS were, median nerve distal sensory latency > $3.4 \mathrm{~ms}$; Median nerve distal motor latency over the thenar $>5.0 \mathrm{~ms}$. Difference between median and ulnar nerve distal sensory latencies $>0.2 \mathrm{~ms}$; Difference between the distal motor latency of median \& ulnar nerve > $1.1 \mathrm{~ms}$; All patients had open carpal tunnel release, intervention for median nerve release using limited uni skin incision technique. Intra-operative measurements of motor distal latency (DML) and proximal latency, amplitude, and conduction velocity and distal sensory latency (DSL), amplitude, and conduction velocity of both the median and ulnar nerves before and after division of the FR 10 to 15 minutes is necessary for Intra operative median nerve conduction study.

Inclusion criteria were: patients with CTS not improve medically. The exclusion criteria were presence of other associated neurological conditions e.g. radiculopathy or peripheral neuropathy, previous CTS release surgery and arthritis of the wrist joints.

Surgical technique: Surgery was performed on the right hand in 28 cases and on the left hand in 8 cases. All patients were operated in the supine position. The affected hand was positioned on the hand-holding of the operating table. The forearm, wrist, and hand were cleaned before surgery with povidone iodine solution to provide sterile surgical conditions. The operation was performed under local anesthesia induced with $2 \%$ ledocaine hydocholoride. No tourniquet was used in any surgery. A longitudinal incision placed along the axis of the radial border of the ring finger, approximately $2 \mathrm{~mm}$ ulnar to the thenar crease, is generally regarded as the safest location for carpal tunnel release incision. The standard longitudinal incision begins distally at Kaplan's cardinal line (which is a line drawn obliquely from the apex of the inter-digital fold between the thumb and index finger distally, toward the ulnar side of the palm parallel to the proximal palmar crease, and passing $4-5 \mathrm{~mm}$ distal to the pisiform) and is extended $2 \mathrm{~cm}$ proximally toward the wrist crease. Where extending the incision proximal to the wrist crease is generally not required in a primary carpal tunnel release. Intraoperative measurements of Motor distal and proximal latency, amplitude, and conduction velocity of both the median and ulnar nerves before and after division of the FR and measurements of distal sensory latency (DSL), amplitude, and conduction velocity of both the median and ulnar nerves were measured before and after division of the FR 10 to 15 minutes is necessary for Intraopera- 
tive median nerve conduction study. After the FR is transected the median nerve completely decompressed from the distal forearm fascia to the superficial palmar arch, the wound is irrigated and hemostasis achieved. The incisions are closed with interrupted mattress sutures (4 - 0 or 5 - 0 non-absorbable material) and covered with a soft dressing and compressive wrap, with careful attention paid to not restrict any digital movement.

Statistical analysis: was done using Statistical Package for Social Sciences (SPSS) VERSION 16. Statistically significant $(\mathrm{p}<0.001)$.

\section{Results}

Our study conducted on 36 wrists in 30 patients (6 cases bilateral) with CTS (Table 1). Twenty three patients were women and 7 patients were men (Table 2), their age ranged from $25-54$ years (mean age $39.5 \pm 2.3$ years) (Table 2). Mean, SD and percentage are shown in (Table 3).

The comparison between preoperative and intraoperative DML distal motor latency of the median nerve after release of the FR showed a statistical significant difference (Figure 1). But DSL and SNCVM showed a statistical non-significant difference as showed in (Table 5).

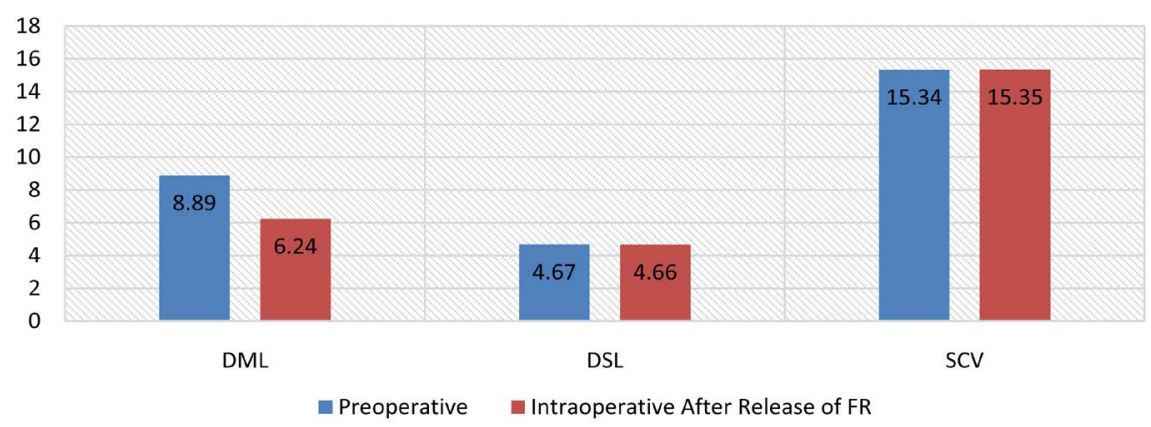

Figure 1. Comparison between preoperative and intraoperative motor and sensory nerve conduction of the median nerve.

Table 1. Age realted to disease duration.

\begin{tabular}{ccccc}
\hline \multirow{2}{*}{ Parameters } & \multicolumn{3}{c}{ Age } & \multicolumn{2}{c}{ Disease duration } \\
\cline { 2 - 5 } & $\mathbf{r}$ & $\mathrm{p}$-Value & $\mathbf{r}$ & $\mathrm{p}$-Value \\
\hline DML & 0.117 & 0.496 & 0.363 & 0.030 \\
DSL & 0.245 & 0.149 & 0.830 & $<0.0001$ \\
SCV & -0.067 & 0.698 & -0.366 & $0.028^{\star}$ \\
\hline
\end{tabular}

Table 2. Sex and occupation relation to disease.

\begin{tabular}{ccccc}
\hline \multirow{2}{*}{ Parameters } & \multicolumn{2}{c}{ Sex } & \multicolumn{2}{c}{ Occupation } \\
\cline { 2 - 5 } & MW & p-Value & MW & p-Value \\
\hline DML & 39 & 0.0232 & 70.5 & 0.0226 \\
DSL & 83 & 0.7414 & 104 & 0.25848 \\
SCV & 68 & 0.32218 & 116 & 0.47152 \\
\hline
\end{tabular}


Table 3. Different parameters mean and SD.

\begin{tabular}{cc}
\hline Patients $(\mathbf{n}=30)$ & Parameters \\
\hline $39.5 \pm 2.3$ & Age, years [Mean \pm SD] \\
\hline $23(76.67 \%)$ & Sex [n (\%)] \\
$7(23.33 \%)$ & Female \\
& Male \\
$19(63.33 \%)$ & Occupation [n (\%)] \\
$11(36.67 \%)$ & Manual worker \\
$6.72 \pm 2.80$ & Office worker \\
& Disease duration, years [Mean \pm SD] \\
$28(93.33 \%)$ & Affected hand \\
$2(6.66 \%)$ & Right \\
$6(20 \%)$ & Left \\
\hline & Bilateral \\
\hline
\end{tabular}

Table 4. Preoperative nerve conduction study.

\begin{tabular}{cc}
\hline & G2 \\
\cline { 2 - 2 } & Mean \pm SD \\
\hline DMLM $(\mathrm{msec})$ & $8.89 \pm 0.93$ \\
DMLU (msec) & $3.0 \pm 0.9$ \\
PMLM (msec) & $7.9 \pm 2.4$ \\
MNCV (M/Sec) & $49.4 \pm 3.2$ \\
DSL M (msec) & $4.67 \pm 0.75$ \\
SNCV M (M/Sec) & $15.34 \pm 3.39$ \\
SNCVU (M/Sec) & $47.3 \pm 2.8$ \\
F-wave M (msec) & $31.2 \pm 3.2$ \\
\hline
\end{tabular}

(DMLM) distal motor latency of the median nerve. (DMLU), distal motor latency of the ulnar nerve. (PMLM) proximal motor latency of the median nerve. (MNCV) median nerve conduction velocity. (SNCVM) sensory nerve conduction velocity of the median nerve. (SNCVU) sensory nerve conduction velocity of the ulnar nerve. (F-wave M F) wave of the median nerve (Table 4).

Table 5. Comparison between preoperative and intraoperative motor and sensory nerve conduction of the median nerve.

\begin{tabular}{ccccc}
\hline & $\begin{array}{c}\text { Preoperative } \\
\text { NCS }\end{array}$ & $\begin{array}{c}\text { Intraoperative after } \\
\text { release of FR }\end{array}$ & P & \% of improvement \\
\hline DML (msec) & $8.89 \pm 0.93$ & $6.24 \pm 1.06$ & $<0.001$ & $29.81 \%$ \\
DSL (msec) & $4.67 \pm 0.75$ & $4.66 \pm 0.76$ & 0.161 & $0.21 \%$ \\
SNCV M (M/Sec) & $15.34 \pm 3.39$ & $15.35 \pm 3.39$ & 0.083 & $0.07 \%$ \\
\hline
\end{tabular}

The motor conduction of median nerve shows dramatic improving during surgery (Figure 1), but the sensory nerve conduction improves mainly after 6 weeks from carpal tunnel surgery (Figure 2) (Table 6). 


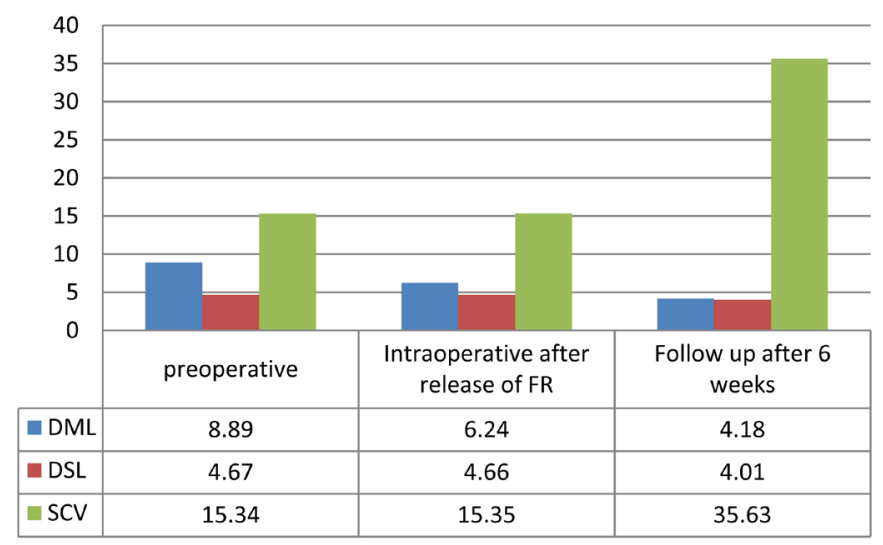

Figure 2. Comparison between intraoperative motor and sensory nerve conduction after complete release of flexor retinaculum (FR) and postoperative after 6 weeks.

Table 6. Comparison between intraoperative motor and sensory nerve conduction after complete release of flexor retinaculum (FR) and postoperative after 6 weeks.

\begin{tabular}{ccccccc}
\hline preoperative & $\begin{array}{c}\text { Intraoperative } \\
\text { after release of FR }\end{array}$ & $\begin{array}{c}\text { Follow up } \\
\text { after 6 weeks }\end{array}$ & F & p & $\begin{array}{c}\text { of } \\
\text { improvement }\end{array}$ \\
\hline DML & $8.89 \pm 0.93$ & $6.24 \pm 1.06$ & $4.18 \pm 0.44$ & 92.91 & $<0.001$ & $52.98 \%$ \\
DSL & $4.67 \pm 0.75$ & $4.66 \pm 0.76$ & $4.01 \pm 0.80$ & 86.21 & $<0.001$ & $14.13 \%$ \\
SCV & $15.34 \pm 3.39$ & $15.35 \pm 3.39$ & $35.63 \pm 4.04$ & 80.32 & $<0.001$ & $132.27 \%$ \\
\hline
\end{tabular}

\section{Discussion}

Treatment of idiopathic CTS ranges from conservative management and/or corticosteroid injection to surgical decompression depending on the degree and the duration of symptoms and etiology of the disease [11]. A total number of 36 wrists in 30 patients ( 6 cases bilateral) with moderate to severe carpal tunnel syndrome were included in our study. Twenty three patients were women and 7 patients were men, their age ranged from 25 - 54 years (with mean age of $39.5 \pm$ 2.3 years) this agrees with Aygul et al., 2005 [12] which means that CTS is a disease of the middle age

Pain along the median nerve distribution was present in all patients $(100 \%)$ and this coincided with Lee et al., 2009 who reported that all patients with symptoms of CTS suffered from pain along distribution of the median nerve [13].

This study showed statistically significant between preoperative and intraoperative median nerve motor latency $\left(\mathrm{p}<0.001^{\star}\right)$ but sensory latency and sensory nerve conduction velocity show non-significant decrease and improve mainly post-operative. This agrees with the results found in a prospective study of 47 patients with 51 hands treated surgically for carpal tunnel syndrome by release of the transverse carpal ligament (TCL) with intraoperative motor nerve conduction latency measured over a standard distance across the carpal tunnel both before and after release of TCL. The results of intraoperative conduction latencies indicated a dramatic and immediate statistically significant reduction 
in the conduction latency across the carpal tunnel in all but seven patients, two of whom had diabetes not show significant results. These data suggest that the rapid decrease in motor conduction latency (DML) due to reversible mechanical or metabolic block, such as ischemia in the segment of the median nerve, may be responsible for the symptoms of carpal tunnel syndrome [14].

This study compared the distal motor and sensory latencies, sensory conduction velocities of the median nerves across the carpal tunnel during surgery this coincided with Taghrid El-Hajj et al., 2010 she reported that compare between the distal motor and sensory latencies, motor and sensory amplitudes, and sensory conduction velocities of the median nerves across the carpal tunnel in patients with the carpal tunnel syndrome preoperatively and postoperatively at 18 and 42 weeks, respectively. The results showed improvement in all the studied variables at 18 weeks after the surgery, except the distal sensory latency improved only at 42 weeks postoperatively [15].

\section{Conclusion}

Intraoperative monitoring of the median nerve shows that the DML rapidly improves than the DSL and SNCV during carpal tunnel surgery. This data support the theory of ischemia due to mechanical block.

\section{Limitations}

The sample size utilized in this study was relatively small. The results might be better established with a larger sample size. In the future, larger populations of CTS patients should be studied.

\section{Conflicts of Interest}

The authors declare no conflicts of interest regarding the publication of this paper.

\section{References}

[1] Klauser, A.S., Halpern, E.J., Faschingbauer, R., et al. (2011) Blind Median Nerve in Carpal Tunnel Syndrome: Assessment with US Cross-Sectional Area Measurement. Radiology, 259, 808-815. https://doi.org/10.1148/radiol.11101644

[2] Demircay, E., Civelek, E., Cansever, T., et al. (2011) Anatomic Variations of the Median Nerve in the Carpal Tunnel: A Brief Review of the Literature. Turkish Neurosurgery, 21, 388-396. https://doi.org/10.5137/1019-5149.JTN.3073-10.1

[3] Bland, J.D. (2001) Do Nerve Conduction Studies Predict the Outcome of Carpal Tunnel Compression? Muscle \& Nerve, 24, 935-940. https://doi.org/10.1002/mus.1091

[4] Kim, J.Y., Kim, J.I, Son, J.E. and Yun, S.K. (2004) Prevalence of Carpal Tunnel Syndrome in Meat and Fish Processing Plants. Journal of Occupational Health, 46, 230-234. https://doi.org/10.1539/joh.46.230

[5] Claes, F., Verhagen, W. and Meulstee, J. (2007) Current Practice in the Use of Nerve Conduction Studies in Carpal Tunnel Syndrome by Surgeons in the Netherlands. Journal of Hand Surgery (European Volume), 32E, 663-667. 
https://doi.org/10.1016/J.JHSE.2007.09.007

[6] Luckhaupt, S.E., Dahlhamer, J.M., Ward, B.W., et al. (2013) Prevalence and Work-Relatedness of Carpal Tunnel Syndrome in the Working Population, United States, 2010 National Health Interview Survey. American Journal of Industrial Medicine, 56, 615-624. https://doi.org/10.1002/ajim.22048

[7] Kostopoulos, D. (2004) Treatment of Carpal Tunnel Syndrome: A Review of the Non-Surgical Approaches with Emphasis in Neural Mobilization. Journal of Bodywork and Movement Therapies, 8, 2-8. https://doi.org/10.1016/S1360-8592(03)00068-8

[8] Ibrahim, I., Khan, W.S., Goddard, N. and Smitham, P. (2012) Carpal Tunnel Syndrome: A Review of the Recent Literature. The Open Orthopaedics Journal, 6, 69-76. https://doi.org/10.2174/1874325001206010069

[9] Bland, J.D. and Rudolfer, S.M. (2003) Clinical Surveillance of Carpal Tunnel Syndrome in Two Areas of the United Kingdom, 1991-2001. Journal of Neurology, Neurosurgery \& Psychiatry, 74, 1674-1679. https://doi.org/10.1136/jnnp.74.12.1674

[10] Vershinin, A.V., Guscha, A.O. and Arestov, S.O. (2018) Surgical Treatment of the Carpal Tunnel Syndrome Using Endoscopic Control and Electrophysiologic Monitoring. Journal of Neurology \& Stroke, 8, 286-288. https://doi.org/10.15406/jnsk.2018.08.00329

[11] Aganval, V., Singh, R., Sachdev, A., et al. (2005) A Prospective Study of the Long-Term Efficacy of Local Methyl Prednisolone Acetate Injection in the Management of Mild Carpal Tunnel Syndrome. Rheumatology, 44, 647-650. https://doi.org/10.1093/rheumatology/keh571

[12] Aygul, R., Ulvi, H., Karatay, S., et al. (2005) Determination of Sensitive Electrophysiologic Parameters at Follow-Up of Different Steroid Treatments of Carpal Tunnel Syndrome. Journal of Clinical Neurophysiology, 22, 222-230.

[13] Lee, J.H., An, J.H., Lee, S.-H., et al. (2009) Effectiveness of Steroid Injection in Treating Patients With Moderate and Severe Degree of Carpal Tunnel Syndrome Measured by Clinical and Electrodiagnostic Assessment. The Clinical Journal of Pain, 25, 111-115. https://doi.org/10.1097/AJP.0b013e3181847a19

[14] Eversmann Jr., W.W., Colonel, L. and Ritsick, J.A. (1978) Intraoperative Changes in Motor Nerve Conduction Latency in Carpal Tunnel Syndrome. The Journal of Hand Surgery, 3, 77-81. https://doi.org/10.1016/S0363-5023(78)80119-1

[15] El-Hajj, T., Tohme, R. and Sawaya, R. (2010) Changes in Electrophysiological Parameters after Surgery for the Carpal Tunnel Syndrome. Journal of Clinical Neurophysiology, 27, 224-226. https://doi.org/10.1097/WNP.0b013e3181dd4ff0 